unerquickliche Zwistigkeiten geführt. Die detaillierten Ausführungen zum Vollzug des Verbots verfassungswidriger Parteien hätten stärker die politische Wirklichkeit berücksichtigen können.

Beide Kommentierungen sind zuverlässig - und überaus gut brauchbar. Die Dichte der Informationen besticht. Wer sucht, wird fündig. Der Nutzen für die Politikwissenschaft wäre bei einer stärkeren Einbeziehung der sozialwissenschaftlichen Erkenntnisse noch größer gewesen. Aber diese Einschränkung tut dem einen wie dem anderen Unterfangen keinen Abbruch.

Eckhard Jesse

\title{
Parlamentarische Kontrolle der Exekutive: verfassungsrechtliche Perspektiven
}

Schmidt, Jörg: Die demokratische Legitimationsfunktion der parlamentarischen Kontrolle (Schriften zum Öffentlichen Recht, Band 1064), Duncker \& Humblot, Berlin 2007, 485 Seiten, $€ 98,-$.

Teuber, Christian: Parlamentarische Informationsrechte. Eine Untersuchung an den Beispielen des Bundestages und des Landtages Nordrhein-Westfalen (Beiträge zum Parlamentsrecht, Band 63), Duncker \& Humblot, Berlin 2007, 336 Seiten, € 69,80.

Christian Teuber und Jörg Schmidt nehmen sich aus ganz unterschiedlichen verfassungsrechtlichen Perspektiven eines aktuellen Problems an: Wie kann parlamentarische Kontrolle angesichts der Privatisierung einstmals staatlicher Tätigkeit gewährleistet werden? Jörg Schmidt wählt den Weg einer rechtsdogmatischen Auseinandersetzung: Im Zentrum stehen dabei nicht einzelne Instrumente parlamentarischer Kontrolle, sondern die grundlegende Frage, welchen Stellenwert die Kontrolle der Exekutive durch das Parlament in der demokratischen Ordnung des Grundgesetzes hat. Der Autor schließt sich zwar der auch in der politikwissenschaftlichen Forschung üblichen Position an, dass es sich um eine selbständige Parlamentsfunktion handelt, die vorrangig in der Kontrolle der Exekutive besteht. Anders als in der üblichen Betrachtung kann parlamentarische Kontrolle hier aber auch in Gesetzesform wahrgenommen werden, ist nicht vor allem Aufgabe der Opposition, die diese öffentlich wahrnimmt, sondern obliegt wesentlich der Mehrheit. In den Worten von Jörg Schmidt ist parlamentarische Kontrolle „zum einen abstrakte Strukturbedingung der demokratischen Legitimation und zum anderen deren konkrete Ausgestaltung“ (S. 121). Im Unterschied zur Gesetzgebung als positiver Legitimation kommt es bei dieser Form der „negativen Legitimation“ staatlichen Handelns nur auf die Möglichkeit zur Einwirkung und nicht auf tatsächliches Kontrollhandeln an. Aus dieser Konzeption von parlamentarischer Kontrolle als Legitimation ergibt sich, dass es für ihr Vorhandensein eines ununterbrochenen Verantwortungszusammenhangs bedarf, bei dem Entscheidungen zugerechnet werden können und Einflussmöglichkeiten bestehen. Indem der Autor dem Begriff der Verantwortung einen solch zentralen Stellenwert einräumt, betont er die klare Verortung seiner Interpretation der grundgesetzlichen Ordnung in der Tradition repräsentativer Demokratie. 
In der Anwendung dieser normativen Konzeption von parlamentarischer Kontrolle auf zwei Bereiche, in denen sie prekär ist, erschließt sich der Nutzen der aufwändigen theoretischen Überlegungen für die Analyse aktuellen demokratischen Regierens auch für einen nicht vorrangig an Rechtsdogmatik interessierten Leser: Anhand der sogenannten ministerialfreien Räume entwickelt Schmidt ein Optimierungsmodell demokratischer Legitimation, das es auch erlaubt, verschiedene Formen von Privatisierung im Hinblick auf ihre Vereinbarkeit mit dem Demokratieprinzip des Grundgesetzes zu bewerten. In ministerialfreien Räumen fehlen die Zurechenbarkeit von administrativen Entscheidungen zum Verantwortungsbereich des Ministers und damit die Grundlage für parlamentarische Kontrolle. In der Diskussion möglicher alternativer Konzeptionen von Legitimation, die das Vorhandensein von Räumen ohne parlamentarische Kontrolle mit dem Demokratieprinzip versöhnen würde, wendet sich Schmidt ausdrücklich gegen die in der Politikwissenschaft häufig herangezogene Output-Legitimation: Demokratie sei ein Formalprinzip und könne sich insofern nur auf die Input-Legitimation stützen (S. 265). Politisch richtige Entscheidung könne mangels eines ex ante bestehenden Konsenses über das Gemeinwohl immer nur die parlamentarisch abgeleitete sein (S. 294). Der von Schmidt entworfene Optimierungsansatz bezieht allerdings die angemessene (effektive) Erfüllung staatlicher Aufgaben, wie sie auch in der Überlegung zur Output-Legitimation eine Rolle spielt, doch wieder ein. Der wesentliche Unterschied liegt darin, dass es beim Optimierungsmodell darauf ankommt, dass staatliche Aufgaben, seien sie nun unmittelbar durch Verfassung oder durch Gesetz begründet, nur dann außerhalb des Regelfalls parlamentarischer Kontrolle erfüllt werden dürfen, wenn das normale Modell parlamentarischer Kontrolle die Aufgabenerfüllung verhindern würde. Es kommt also darauf an, eine ,individuelle, aufgaben- und legitimationsangemessene Struktur" (S. 367) zu finden und von Seiten des Parlaments stets zu prüfen, ob die Entscheidung für die Ausgliederung aus dem parlamentarischen Verantwortungszusammenhang richtig und notwendig war.

Im Kontext der Anwendungsfelder greift Schmidt auch konkrete Formen parlamentarischer Kontrolle wie Frage- und Interpellationsrechte, Berichtspflichten sowie Untersuchungsausschüsse auf. Diese Ausführungen bleiben jedoch sehr knapp, was die Grenzen der Untersuchung verdeutlicht: Vorrangiges Interesse sind rechtsdogmatische Fragen; die realen Grenzen parlamentarischer Einwirkungsmöglichkeiten auf das Handeln der Exekutive sind dem Autor zwar bewusst und werden punktuell auch thematisiert, stehen jedoch nicht im Zentrum der Arbeit.

Auch in Christian Teubers Untersuchung stehen vom ihm mehrfach als Forschungsdesiderat beschriebene politikwissenschaftliche Fragen des Parlamentsalltags nicht im Mittelpunkt. Anders als Schmidt interessiert er sich für die konkreten Instrumente, die den Parlamenten in Deutschland zur Beschaffung von Informationen zur Verfügung stehen. Differenzen gibt es zwischen den beiden Studien nicht nur in der thematischen Ausrichtung, sondern auch in der theoretischen Grundlegung. Teuber leitet unmittelbar aus Artikel 20 GG ein umfassendes parlamentarisches Informationsrecht ab. Nur wenn das Parlament über das Handeln der Exekutive informiert sei, könne „inhaltliche Rückkopplung der Ausübung staatlicher Gewalt an den parlamentarischen Willen" stattfinden und so die vom Bundesverfassungsgericht „geforderte(.) ununterbrochene(.) Legitimationskette“ hergestellt werden (S. 45). Die Notwendigkeit, dass das Parlament informiert ist, ergibt sich dabei nicht nur aus der Funktion parlamentarischer Kontrolle der Exekutive, sondern ebenso aus der Gesetzgebungsfunktion sowie in geringerem Maße aus der Kreationsfunktion. Die Ein- 
rede eines Kernbereichs der exekutiven Verantwortung, der parlamentarischer Information nicht zugänglich sein dürfe, lässt Teuber nur in sehr begrenztem Umfang gelten. Hier unterscheidet er sich von Schmidt, der umfassende parlamentarische Informationsrechte unter Verweis auf den notwendigen Eigenbereich der Exekutive als Bestandteil des Systems von Checks and Balances, wie er es im Grundgesetz verwirklicht sieht, ablehnt.

Teuber entwickelt ein abgestuftes System parlamentarischer Selbst- und Fremdinformationsrechte, deren Umfang danach variiert, auf welche Parlamentsfunktion sie sich beziehen. Im Kontext der Privatisierung von Staatsaufgaben ist nun in besonderer Weise interessant, inwiefern sich parlamentarische Informationsrechte auch auf das Handeln privater Akteure erstrecken. Dazu bedarf es eines erweiterten Begriffs von Staatsgewalt, der nicht allein an dem formalen Kriterium des Handelns staatlicher Organe anknüpft. Teuber liefert dazu nur eine knappe Definition, während Schmidt das Problem ausführlicher aus rechtsdogmatischer Sicht diskutiert. Im Ergebnis kommen beide zu einem erweiterten Verständnis von Staatstätigkeit, das nicht an der Grenze der staatlichen Organe Halt macht. Auf dieser Grundlage diskutiert Teuber die Reichweite des parlamentarischen Informationsrechts anhand konkreter Instrumente, die sich aus dem Grundgesetz, der Nordrhein-Westfälischen Landesverfassung, haushaltsrechtlichen Regelungen sowie weiteren gesetzlichen Bestimmungen ergeben.

Die unterschiedlichen Herangehensweisen führen auch im Konkreten zu divergierenden Resultaten: Teuber verhilft durch die am Maßstab eines umfassenden Informationsrechts des Parlaments gewonnene Auslegung aktien- und gesellschaftsrechtlicher Vorschriften der parlamentarischen Kontrolle auch in Bereichen privatisierter Staatstätigkeit zur Durchsetzung. Schmidt hingegen sieht diesen Ausweg nicht, sondern stellt rechtlichen Gestaltungsbedarf fest, damit den Anforderungen parlamentarischer Kontrolle Genüge getan werden kann. Für die gegenwärtige Lage kommt er zu dem Ergebnis, „daß die Privatisierungspraxis der vergangenen Jahre den demokratiestaatlichen Anforderungen weithin nicht entspricht“ (S. 420). Eine eher kritische Einschätzung der gegenwärtigen Lage wird auch bei Teuber deutlich, der an verschiedenen Stellen die Auffassung vertritt, dass die geltenden Regelungen dem verfassungsrechtlich fundierten Informationsanspruch des Parlaments nicht gerecht werden.

Auf je eigene Art zeigen beide Studien, dass mit der zunehmenden Privatisierung von staatlichen Aufgaben grundlegende Fragen demokratischer Legitimation und parlamentarischer Kontrolle berührt sind. Die von der üblichen politikwissenschaftlichen Betrachtung abweichende Konzeptionierung der Kontrolle im Kontext der Parlamentsfunktionen sowie die Ausführungen zu Fragen demokratischer Legitimation und Legitimität sind durchaus geeignet, üblich gewordene politikwissenschaftliche Begrifflichkeit aus einer normativen, verfassungstheoretischen Perspektive auf den Prüfstand zu stellen. Mit der genauen Bestandsaufnahme der Kontrollinstrumente ist eine Grundlage für weitergehende Untersuchungen geschaffen, wie diese Instrumente in der parlamentarischen Praxis wirken. Für die politikwissenschaftliche Parlamentarismusforschung bleibt in dem hier theoretisch eröffneten Feld viel zu tun. 